\title{
Performance Characterization and Improvement for CCD Cameras in Transmission Electron Microscopy
}

\author{
Kenneth H. Downing
}

Life Sciences Division, Lawrence Berkeley National Laboratory, Berkeley, CA 94720

The development of charge coupled device (CCD) cameras for the consumer market has been dramatic over the last several years. A significant fraction of the population now uses inexpensive cell phones that have built-in cameras with better resolution than was recently available for electron microscopy at hundreds of times the price. While there have been tremendous advances in the technology for producing many types of CCD chips, the requirements for effective performance in microscopy have so far made it impractical to take advantage of the mass produced chips. Still, CCDs are now in routine use for a number of TEM applications, particularly where large series of images are needed such as in tomography and wavefront reconstruction from focus series, and where the benefits of rapid, digital data recording outweigh any of the disadvantages in CCD performance compared to other data recording media. We discuss here some of the performance issues that relate particularly to high-end cameras and how limitations in the performance might be overcome.

All CCD cameras used with electron microscope currently use a scintillator to convert the electron beam energy to photons which are then passed to the light-sensitive CCD chip. The signal is read out through a series of amplifiers. CCDs have the very favorable property of a highly linear response - the output signal is proportional to the electron intensity over a wide rang of intensities. Ideally this linearity would extend to very low intensities so that every single electron would be clearly detected. A number of factors combine to make the signal from a single-electron somewhat difficult to detect. The electron-to-photon conversion generally utilizes only a fraction of the electron energy. Some fraction of the resultant photons is lost before reaching the CCD. The conversion of photons to signal in the CCD is less than $100 \%$ efficient. Finally there is always some noise introduced in the readout process.

The amount of noise in an electronic circuit decreases as the bandwidth of the circuit decreases. In the context of CCD readout, this means that we can greatly reduce the readout noise by reading the signal from the CCD slowly. Thus the CCDs used for most high quality data collection are "slow scan" CCDs. The slow readout also improves the efficiency of the internal charge transfers involved in extracting the signal. As for the light conversion efficiency of the CCD, manufacturers have steadily been making improvements that lead to better sensitivity and spectral response. Developments in these areas have been effectively driven at least as much by astronomers as by electron microscopists; while the number of high-end telescopes is much smaller than for electron microscopes, the total costs involved are much greater, providing a funding source for the very expensive developments in the technology.

The interaction of the electrons with the scintillator and subsequent coupling to the CCD has been an area of very active development by the microscopy community. The scintillator is most commonly attached to a fiber optic plate that is in turn bonded to the CCD chip. Tapered fiber optics can change the size scale or magnification between the scintillator and the CCD pixels over a 
small range, but to a good approximation the image in the scintillator is projected directly to the CCD. This has turned out to be a problem of increasing significance as the accelerating voltage of microscopes has increased to around $300 \mathrm{kV}$. At this voltage the point spread function (PSF) of the scintillator, or the area which produces light from a single electron, increases because the electrons scatter within the scintillator, and many pass through it and then scatter within the fiber optic and back into the scintillator. In addition, only a small part of the electron energy is converted to photons at the higher voltage, and the number of photons produced per electron becomes more random as more of the electrons pass through the scintillator. One might overcome this problem by simply making the scintillator thicker, but this increases the width of the PSF, which is already larger than the pixel size.

At the same time that microscopists are tending toward higher voltages, the CCD manufacturers are tending toward making the pixels smaller. Making the pixels smaller provides advantages in speed and other performance criteria, and allows for an increase in the number of pixels on the chip. However, it aggravates the mismatch between their size and the PSF. Thus there is a serious need to develop ways to improve the coupling of the scintillator and the CCD. Two approaches currently under development are sketched in Figure 1.

Placing the scintillator on an electron-transparent film avoids the backscatter from the fiber optic, and at the same time allows the use of a lens to couple to the CCD. An optical demagnification can be used to reduce the image so that the scintillator PSF matches the pixel size. This allows a better choice of scintillator thickness to optimize the light output, although most $300 \mathrm{kV}$ electrons still pass through.

We have taken a complementary approach in which we reduce the energy of the electrons before they impact the scintillator. The CCD, with conventional fiber-optically coupled scintillator, is mounted below a decelerator tube and floated to a potential that can be a few tens of $\mathrm{kV}$ below the accelerating voltage. This arrangement provides several advantages, including reducing the PSF to an almost arbitrarily small size and capturing all of the electron energy within the scintillator.

Prototypes of both of these systems have been built and are being characterized. There are still engineering challenges to be solved in each system, so the relative merits are, at this moment, not entirely clear. It is clear, though, that we can expect continued and substantive improvement in the capabilities of CCD cameras applied to electron microscopy.

Figure 1. Two approaches to obtaining better CCD performance. At left, a lens is used to optically couple the scintillator (scint) output to the CCD. At right, the electrons pass through a retarding electric field in a decelerator tube (DEC) before hitting the scintillator mounted on a fiber optic plate (FO)

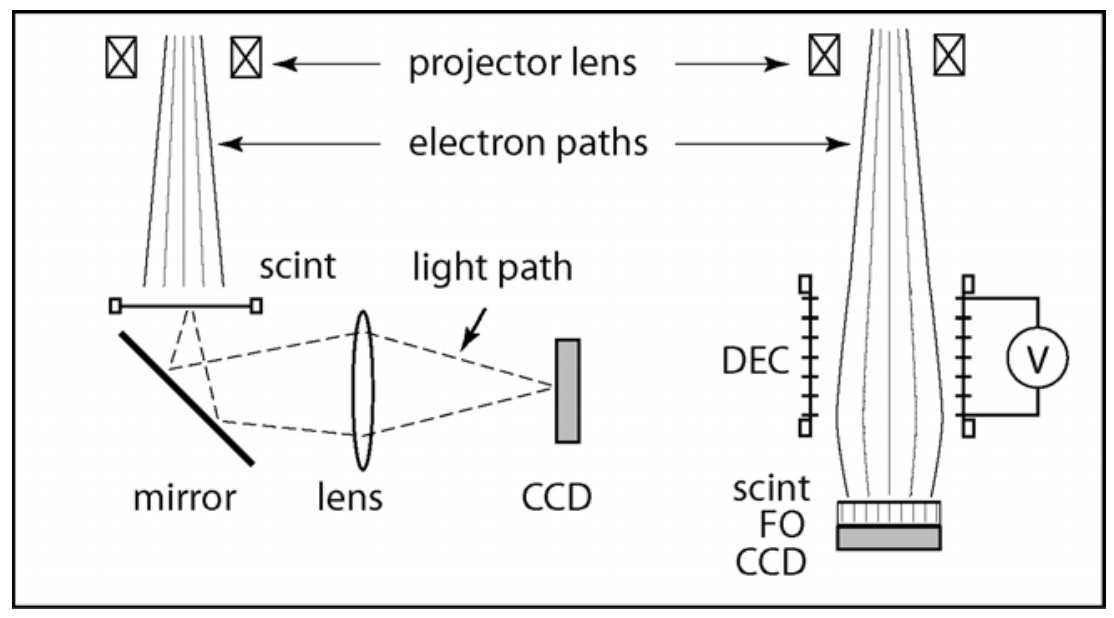

